\title{
OPTICAL DOPING OF NITRIDES BY ION IMPLANTATION
}

\author{
E.ALVES* \\ Instituto Tecnológico e Nuclear, EN10, 2686-953 Sacavém, Portugal \\ K. LORENZ, R.VIANDEN \\ Institut für Strahlen- und Kernphysik, Univ. Bonn, Nussallee \\ 14-16, D-53115 Bonn, Germany \\ C. BOEMARE, M. J. SOARES, T. MONTEIRO \\ Departamento de Física, Universidade de Aveiro, 3810 Aveiro, Portugal
}

\begin{abstract}
A series of rare earth elements (RE) were implanted in GaN epilayers to study the lattice site location and optical activity. Rutherford backscattering spectrometry in the channeling mode(RBS/C) was used to follow the damage behavior in the Ga sublattice and the site location of the RE. For all the implanted elements (Ce, $\mathrm{Pr}, \mathrm{Dy}, \mathrm{Er}$, and $\mathrm{Lu}$ ) the results indicate the complete substitutionality on Ga sites after rapid thermal annealing at $1000^{\circ} \mathrm{C}$ for $2 \mathrm{~min}$. The only exception occurs for Eu that occupies a Ga displaced site. Annealing at $1200^{\circ} \mathrm{C}$ in nitrogen atmosphere at a pressure of $1 \mathrm{GPa}$ is necessary to achieve the complete recovery of the damage in the samples. After annealing the recombination processes of the implanted samples were studied by above and below band gap excitation. For Er implanted samples besides the $1.54 \mu \mathrm{m}$ emission green and red emissions are also observed. Red emissions from ${ }^{5} \mathrm{D}_{0} \rightarrow{ }^{7} \mathrm{~F}_{2}$ and ${ }^{3} \mathrm{P}_{0} \rightarrow{ }^{3} \mathrm{~F}_{2}$ transitions were found in $\mathrm{Eu}$ and $\mathrm{Pr}$ implanted samples even at room temperature.
\end{abstract}

\section{Introduction}

Rare earth (RE) doped GaN is of considerable interest due to its large application potential in optoelectronic devices. RE elements have a partially filled inner $4 f^{\mathrm{n}}$ shell, which is shielded, from the surroundings by completely filled outer shells leading to very sharp optical emission lines in the visible and infrared region. For GaN it was shown that, in contrast with semiconductors with narrow bandgaps ( $\mathrm{Si}$, GaAs etc.), the temperature quenching is much lower and the solubility of the RE atoms is higher. ${ }^{1} \mathrm{~A}$ large number of studies were reported about the photoluminescence and cathodoluminescence mainly of samples doped during growth with various RE elements. ${ }^{2}$ Electroluminescent devices from excited RE states were obtained covering the entire visible spectrum: light emission in the green $(\mathrm{Er})^{3}$, yellow/orange $(\mathrm{Er}$ and $\mathrm{Eu})$ ${ }^{4}$ red $(\mathrm{Eu} \text { and } \mathrm{Pr})^{5,6}$ and blue $(\mathrm{Tm})^{7}$. However the incorporation of the large concentration needed to reach the high intense emissions is limited by the interaction of the dopants during the growth process. Ion implantation represents an alternative method to introduce the RE atoms allowing for optical selective-area doping. However the incorporation of ion implantation on the GaN processing techniques relies on the possibility to anneal the radiation damage and incorporation of the RE into optical active sites.

In this article we report RBS/channeling measurements to study the damage recovery and determine the lattice site of several RE elements into GaN. The optical activation of The RE after annealing was investigated by PL measurements. The excited states of the optical centers induced by rare earth implantation were characterised.

\section{Experimental Details}

Several RE elements were implanted into GaN epilayers grown by Metal Organic Chemical Vapor Deposition on c-plane sapphire. The thickness of the nominally undoped $\left(\mathrm{n}<5 \times 10^{16} \mathrm{~cm}^{-3}\right) \mathrm{GaN}$ films was $1.3 \mu \mathrm{m}$. The RE elements Ce, Pr, Eu, Dy, Er and $\mathrm{Lu}$ were implanted at room temperature with an energy of $160 \mathrm{keV}$ and doses of $5 \times 10^{14} \mathrm{~cm}^{-2}$ and $5 \times 10^{15} \mathrm{~cm}^{-2}$. The measured implantation ranges were determined to lie

* Corresponding author: Dep. Física, Instituto Tecnológico e Nuclear, EN.10 2686-953 Sacavém ,Portugal 
between 30 and $40 \mathrm{~nm}$ in good agreement with TRIM99 simulations. The samples were annealed in a rapid thermal annealing apparatus between graphite strips under flowing $\mathrm{N}_{2}$ gas at $1000^{\circ} \mathrm{C}$ for $120 \mathrm{~s}$.

$\mathrm{RBS} /$ channeling studies were performed with a $1 \mathrm{~mm}$ diameter collimated beam of 2 $\mathrm{MeV} \mathrm{He}{ }^{+}$ions. The backscattered particles were detected at $140^{\circ}$ and close to $180^{\circ}$ with respect to the incoming beam direction using silicon surface barrier detectors with resolutions of 13 and $16 \mathrm{keV}$, respectively, located in the standard IBM geometry. Full angular scans were carried out across the $\langle 0001\rangle$ and the $\langle 10-11\rangle$ axial directions using a two-axis goniometer. To reduce the pile-up effect, which would degrade the sensitivity for the rare earth signal, the beam current was kept below 2 nA. Computer simulations were performed using a Monte Carlo code where the wurtzite structure was incorporated in order to simulate the $\mathrm{GaN}$ angular scan. ${ }^{8}$

Photoluminescence measurements in the visible spectral region were carried out with a $325 \mathrm{~nm} \mathrm{CW} \mathrm{He-Cd} \mathrm{laser} \mathrm{and} \mathrm{the} \mathrm{excitation} \mathrm{power} \mathrm{density} \mathrm{was} \mathrm{typically} \mathrm{less} \mathrm{than} 0.6$ W.cm ${ }^{-2}$. PL was measured at temperatures between $14 \mathrm{~K}$ and $300 \mathrm{~K}$ using a closed cycle helium cryostat. The luminescence was dispersed by a Spex 1704 monochromator $(1 \mathrm{~m}$, $1200 / \mathrm{mm}$ ) and detected by a Hamamatsu R928 photomultiplier. The details for infrared measurements were published elsewhere. ${ }^{9}$

\section{Results and Discussion}

\subsection{RBS/channeling}

Figure 1 presents the random and the <0001> aligned RBS spectra of the samples implanted with two different doses of $\mathrm{Pr}$.

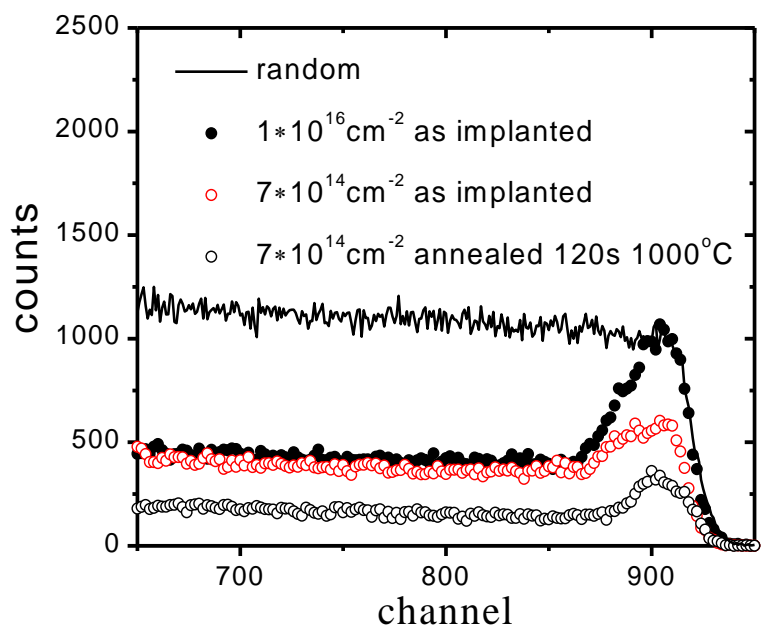

Fig.1. Random and <0001> aligned RBS spectra obtained for GaN implanted with Pr with two different implanted doses. For the lower dose the spectrum after rapid thermal annealing at $1000^{\circ} \mathrm{C}$ are shown.

For the high dose only the as implanted spectrum is shown whereas for the low dose spectra are shown before and after annealing at $1000^{\circ} \mathrm{C}$ for $120 \mathrm{~s}$. For the high dose it can be seen that the backscattering yield in the implanted region reaches the same values as the random spectrum. This is an indication that amorphisation of this surface layer occurred. Damage is below the amorphisation threshold for the lower dose. After annealing at $1000^{\circ} \mathrm{C}$ the lattice damage anneals out to a big extent for the sample 
implanted with the lowest dose. This type of behavior was observed for all the implanted samples. In the case of Er the complete recovery was only achieved after annealing at $1200^{\circ} \mathrm{C}$ for 20 min under a nitrogen atmosphere at $1 \mathrm{GPa} .^{10}$

Angular scans through the $<0001>$ axis for all the implanted samples with the low dose, directly after implantation, show minimum yields between 30 and 50\%. For the determination of these yields the window for Ga was set from 20 to $80 \mathrm{~nm}$ containing all the implanted atoms. After annealing the minimum yield decreased to values between 15 and $30 \%$ reflecting the damage recovery. The corresponding RE scans show a complete overlap with the Ga scans for all analyzed elements before and after annealing indicating that both the RE and the Ga sublattice is affected to an equal extent by the disorder caused by the implantation. Furthermore the overlap indicates the shadowing of the implanted ions along the $<0001>$ direction. However Ga and $\mathrm{N}$ form mixed rows along the <0001> axis and thus it is not possible to determined unambiguously the lattice site of the RE. Scans across the $\langle 10-11\rangle$ axis where done for this purpose since here it is possible to distinguish between $\mathrm{Ga}$ and $\mathrm{N}$ sites (see the projections on figure 3 ). Due the large difference in the atomic numbers of $\mathrm{Ga}$ and $\mathrm{N}$ the steering potentials lead to different widths of the angular scans. ${ }^{9}$ The results in figure 2 shows an example of the scans through the $\langle 0001\rangle$ and the $\langle 10-11\rangle$ after annealing for the Pr implanted sample. The good overlap of the scans for Ga and Pr for the two scans proves that $\mathrm{Pr}$ is completely incorporated into $\mathrm{Ga}$ sites. The fraction of substitutional atoms does not change after annealing.
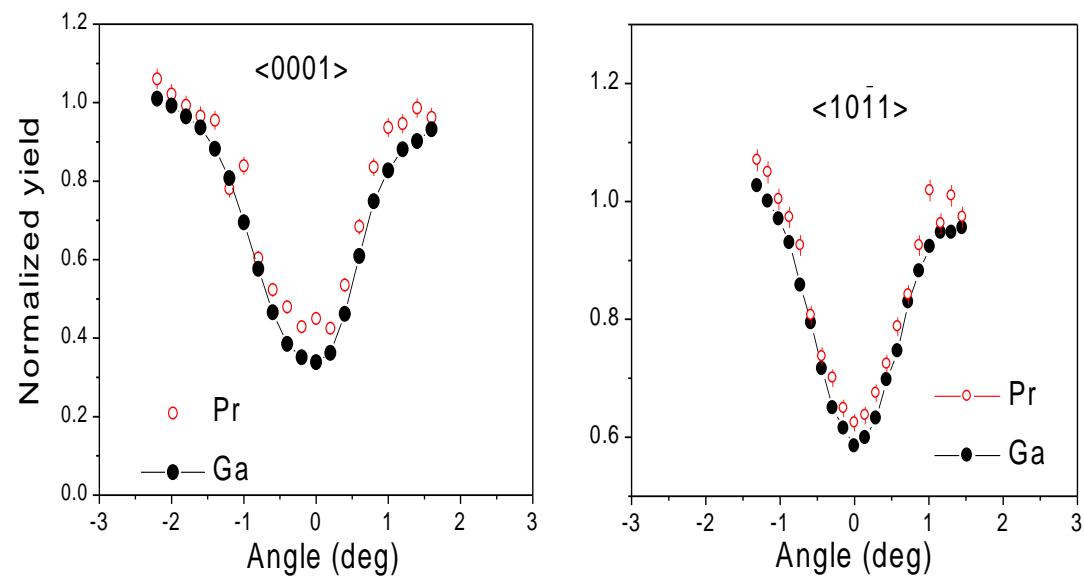

Fig. 2. Angular scans across the $<0001>$ and $<10-11>$ axes for the sample implanted with Pr after annealing at $1000^{\circ} \mathrm{C}$ for $120 \mathrm{~s}$.

This result is in agreement with the work of U.Wahl et al. who found a large substitutional fraction for very low doses of Pr in GaN using the emission channeling technique. ${ }^{11}$ The results obtained for Ce, Dy, Er and Lu were essentially the same. Monte Carlo simulations yield substitutional fractions between 90 and $100 \%$ for all these RE. The only exception occurs for Eu. In fact, while for the $<0001>$ axis the Eu and $\mathrm{Ga}$ scans overlap, like for the other RE, the scans across the $\langle 10-11\rangle$ axis reveal different widths as shown in figure 3 . In this case the data was simulated assuming a displacement of the $\mathrm{Eu}$ atom from the $\mathrm{Ga}$ site of $0.2 \AA$ along the c-axis. This 
displacement could be related with the larger ionic radius of $\mathrm{Eu}(1.12 \AA)$ when compared with the other RE ions.
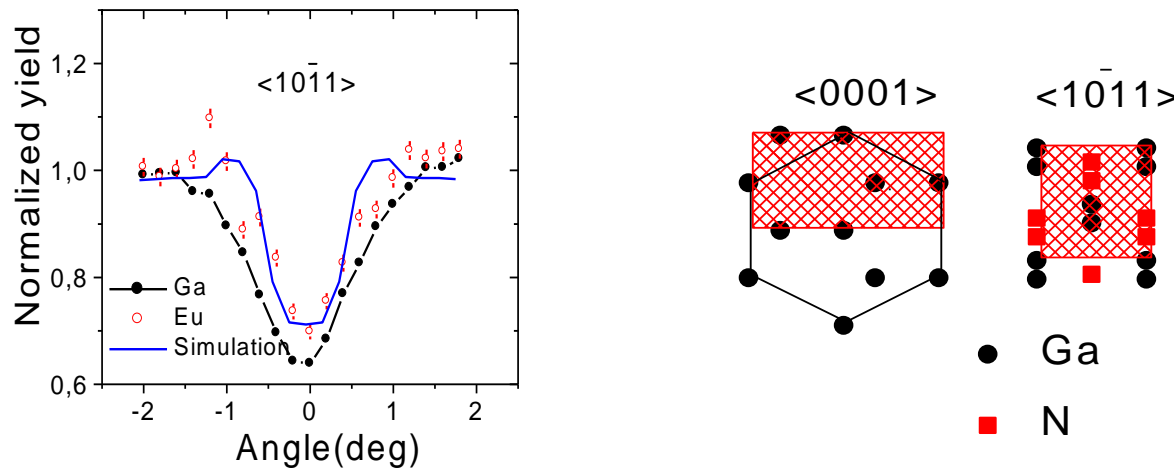

Fig. 3. Angular scan across the $<10-11\rangle$ axis for the sample implanted with Eu at a dose of $5 \times 10^{14} \mathrm{~cm}^{-2}$. The fit obtained with the Monte Carlo simulation is also shown. The projections show the positions of the $\mathrm{Ga}$ and $\mathrm{N}$ lattice sites along the directions shown. The shadowed areas are the cells used in the simulations.

\subsection{Photoluminescence}

The PL spectra of the strongest emission lines for Pr and Eu implanted GaN samples are shown in figure 4. The PL spectra was obtained by above band gap excitation at 14 K.The ${ }^{5} \mathrm{D}_{0} \rightarrow{ }^{7} \mathrm{~F}_{2}$ transition are the strongest emission lines either at $14 \mathrm{~K}$ and room temperature for the Eu doped samples. The minor lines observed in the studied spectral range were tentatively assigned to different ${ }^{5} \mathrm{D}_{0,1} \rightarrow{ }^{7} \mathrm{~F}_{0,1,2,3,4}$ transitions in accordance

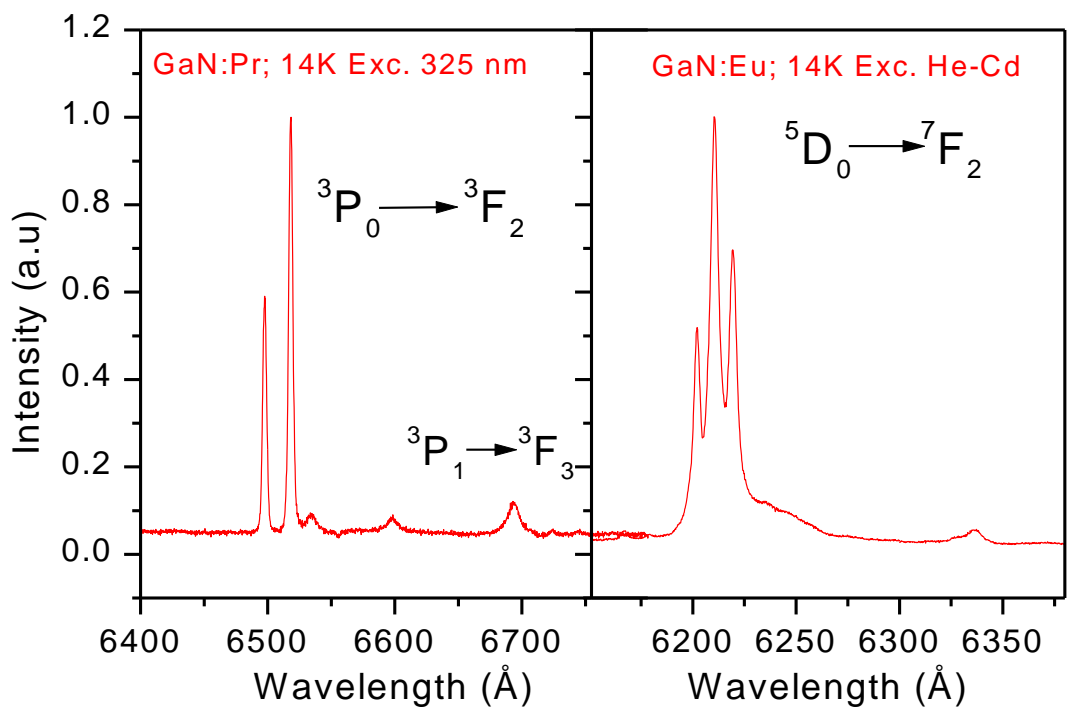

Fig.4. PL spectra of Pr and Eu implanted GaN sample obtained by He-Cd excitation at $14 \mathrm{~K}$.

with previous results for the $\mathrm{Eu}^{3+}$ in several hosts. ${ }^{12}$ The high resolved PL spectra put in evidence that the ion must be in lower site symmetry than the refereed trigonal symmetry $\left(\mathrm{C}_{3 \mathrm{~V}}\right)^{13}$ being $\mathrm{D}_{2}$ point group tentatively assigned to the ion site. ${ }^{12}$ The 
displacement from the regular $\mathrm{Ga}$ site found for Eu ion fully agrees with the PL findings. On the other hand for Pr the strongest emission lines are identical to that ones previously observed by several authors for the ${ }^{3} \mathrm{P}_{0} \rightarrow{ }^{3} \mathrm{~F}_{2}$ transition indicating that $\operatorname{Pr}^{3+}$ ion is in $\mathrm{C}_{3 \mathrm{v}}$ symmetry in agreement with our lattice site location results. ${ }^{14}$ In a similar way to the $\mathrm{Eu}^{3+}$ luminescence the integrated intensity dependence on temperature of the ${ }^{3} \mathrm{P}_{0} \rightarrow{ }^{7} \mathrm{~F}_{2}$ transition obtained under constant illumination decreases near one order of magnitude in the studied temperature range $(14 \mathrm{~K} \text { to } \mathrm{RT})^{12}$.

Also PL studies in Er implanted samples were performed and besides the characteristic near infrared luminescence at $1.543 \mu \mathrm{m}$, green and red luminescence was observed. ${ }^{9} \mathrm{PL}$ studies of $\mathrm{Dy}, \mathrm{Lu}$ and $\mathrm{Ce}$ are under way and preliminary results points to an optical activation of the RE ions.

\section{Conclusions}

Our results show that the RE's Ce, Dy, Pr, Er and $\mathrm{Lu}$ implanted in GaN were incorporated into the $\mathrm{Ga}$ lattice site of the structure. The implantation damage is partially recovered during rapid thermal annealings at $1000^{\circ} \mathrm{C}$ for $120 \mathrm{~s}$. For $\mathrm{Er}$ implantation the anneling at $1200^{\circ} \mathrm{C}$ under an overpressure of nitrogen was enough to recrystallize completely the damage region. Contrary to the other RE, Eu occupies a site displaced from the regular $\mathrm{Ga}$ site. According with the Monte Carlo simulation a displacement of $0.2 \AA$ along the $<0001>$ direction reproduces our results. The PL results also agree in lower site symmetry than trigonal $\mathrm{C}_{3 \mathrm{v}}$ symmetry for $\mathrm{Eu}^{3+}$. For Pr implanted samples the PL data pointed to a substitutionality of the ion in Ga sites. For both implanted ions a decrease of near one order of magnitude for the integrated intensity dependence on temperature is observed in the range of $14 \mathrm{~K}$ to room temperature.

\section{Acknowledgement}

We acknowledge the .......

\section{References}

1. A.J. Steckl, J.M. Zavada, MRS Bull. 24, (1999) 33

2. A.J. Steckl, J. Heikenfeld, M. Garter, R. Birkhahn, D.S. Lee, Compound Semiconductor 6 (2000) 48

3. A. J. Steckl, M. Gartner, R. Birkhahn, J. Scofield, Appl. Phys. Lett 73 (1998) 2450.

4. D. S. Lee, J. Heikenfeld, R. Birkhahn, M. Garter, B. K. Lee, A. J. Steckl, Appl. Phys. Lett., 76 (2000) 1525.

5. R. Birkhahn, M. Garter, A. J. Steckl, Appl. Phys. Lett., 74 (1999) 2161.

6. J. Heikenfeld, M. Gartner, D. S. Lee, R. Birkhahn, A. J. Steckl, Appl. Phys. Lett., 75 (1999) 1189.

7. A. J. Steckl, M. Gartner, D. S. Lee, J. Heikenfeld, R. Birkhahn, Appl. Phys. Lett., 75 (1999) 2184.

8. E. Alves, M.F. da Silva, J.C. Soares, J. Bartels, R. Vianden, C.R. Abernathy, S.J. Pearton, MRS Internet J. Nitride Semicond. Res. 4S1, G11.2 (1999)

9. T. Monteiro, J. Soares, M. R. Correia, E. Alves, J. Appl. Phys., 89 (2001) 6183

10. 10.E. Alves, T. Monteiro, J. Soares, L. Santos, M.F. da Silva, J.C. Soares, W. Lojkowski, D.Kolesnikov, R. Vianden, J.G. Correia, Mat. Sci. and Eng. B81 (2001) 132

11. U. Wahl, J. P. Araújo, L. Peralta, J. G. Correia and ISOLDE Collaboration, J. Appl. Phys., 88 (2000) 1319

12. T. Monteiro, C. Boemare, M. J. Soares, R.A.Sá Ferreira, L.D. Carlos, K. Lorenz, R. Vianden E. Alves, Physica B (submitted)

13. H. J. Lozykowski, W. M. Jadwisienczak, J. Han, I. G. Brown, Appl. Phys. Lett., 77 (2000) 767. 
14. J. M. Zavada, R. A. Mair, C. J. Ellis, J. Y. Lin, H. X. Jiang, R. G. Wilson, P. A. Grudowski, R. D. Dupuis, Appl. Phys. Lett., 75 (1999) 790. 